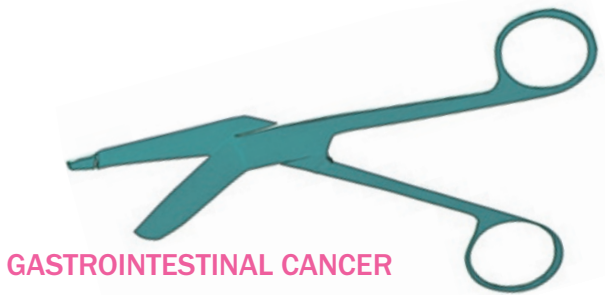

\title{
Less is more for surgical treatment of oesophageal cancer
}

In the past two decades, the worldwide incidence of oesophageal cancer has increased considerably, with over 480,000 new diagnosed cases recorded in 2008. Although the mortality rate from oesophageal cancer is low, at least half of patients who are treated with open surgery are at risk of developing pulmonary complications, which increases hospital stay duration and affects patient quality of life.

The need for open surgery can be avoided by minimally invasive oesophagectomy, which reduces rates of pulmonary infection and hospital stay. However, no randomized clinical trials have compared the benefits of open surgery with minimally invasive oesophagectomy. Now, a study led by Miguel Cuesta has provided the first clear evidence of the shortterm benefits of minimally invasive oesophagectomy for patients with operable oesophageal cancer.
In this multicentre, open-label randomized trial carried out in five European centres, 115 patients were randomly assigned to receive either open oesophagectomy $(n=56)$ or minimally invasive oesophagectomy $(n=59)$. The primary outcome was postoperative pulmonary infection and secondary outcomes included length of hospital stay, quality of life assessment and other postoperative complications.

The results of the trial were encouraging: in the first 2 weeks after surgery, significantly fewer patients in the minimally invasive surgery group had pulmonary infections compared with the open surgery patients (29\% versus 9\%). Furthermore, fewer patients in the minimally invasive surgery arm contracted a pulmonary infection. Given these data it is not surprising that hospital stay was also considerably shorter (11 versus 14 days) for those in the minimally invasive surgery group compared with the open surgery cohort. Quality of life was also better for patients receiving minimally invasive surgery than those treated with open surgery. Other post operative complications did not differ significantly between the two groups. The researchers comment "importantly, we noted no compromise in the quality of the resected specimen and no significant differences in the number of lymph nodes retrieved. Long-term outcome measurements, including survival analyses, are planned for the future."

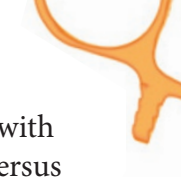

Lisa Hutchinson

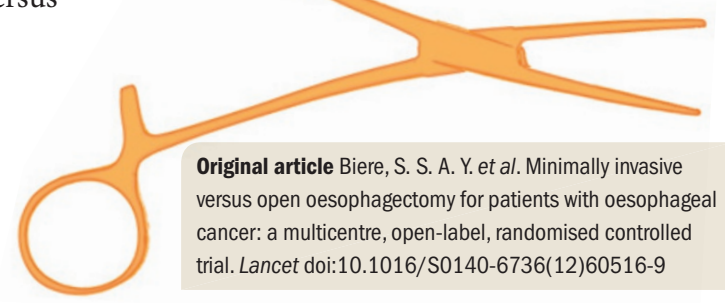

